\title{
EFFECT OF CATION SUBSTITUTION IN "123"-TYPE SUPERCONDUCTING OXIDES ON POWDER DIFFRACTION INTENSITIES
}

\author{
W. Easocha, S.A. Hodorowicz, A. Chodorowicz-Bąk \\ Faculty of Chemistry, Jagiellonian University, Karasia 3, 30-060 Kraków, Poland \\ AND H.A. EICK \\ Michigan State University, East Lansing, MI 48824, USA \\ (Received July 29, 1991; in final form February 13, 1992)

\begin{abstract}
This research was undertaken to determine whether by powder diffraction substitution in the "123"-type high $T_{\mathrm{c}}$ oxides, $\mathrm{Ln}_{1-x} \mathrm{M}_{x} \mathrm{Ba}_{2-y} \mathrm{M}_{y} \mathrm{Cu}_{3-z}$ $\mathrm{M}_{z} \mathrm{O}_{6.5+\delta}$ (where $\mathrm{Ln}=$ lanthanoid, $\mathrm{M}_{x}=\mathrm{Me}^{3+}, \mathrm{M}_{y}=\mathrm{Me}^{2+}$ and $\mathrm{M}_{z}=$ $\mathrm{Me}^{3+}$ and $\mathrm{Me}^{2+}$ ) could be detected unequivocally. Numerous X-ray and a few neutron powder diffractograms were calculated for substituted compounds. Reflections most sensitive to substitution and the influence of site occupancy for $\mathrm{Cu}$ and $\mathrm{O}$ atoms are also characterized. The results are compared with some experimental data reported in the literature.
\end{abstract}

PACS numbers: 74.70.Vy

\section{Introduction}

Synthesis of materials related to the high $T_{\mathrm{c}}$ superconducting oxides in an effort to identify new materials with improved superconducting parameters has been exceedingly active research area. Compounds based on the "123"-type superconductor have been synthesized frequently by substituting related elements for $\mathrm{Ln}, \mathrm{Ba}$ and $\mathrm{Cu}[1-6]$. While analytical data can suggest strongly that substitution has occurred, only a crystal structure determination provides unquestionable proof. However, for a structural investigation a good quality single crystal must be obtained. In some cases the need for a single crystal could be avoided if powder diffraction data and the Rietveld refinement procedure were used. The Rietveld procedure is particularly useful for determination the position and occupancy of oxygen sites when used with neutron powder diffraction data.

In contrast to neutron diffraction equipment, X-ray powder diffraction equipment is generally available, and the method is "cost effective". This work was 
undertaken to establish the extent to which substitution and/or occupancy parameters can be determined in " 123 "-related oxides by the X-ray powder "integrated intensity methods". The results are compared to selected literature reports in which substitution was substantiated by powder diffraction data.

\section{Calculation details}

Atomic positional and thermal parameters of $\mathrm{YBa}_{2} \mathrm{Cu}_{3} \mathrm{O}_{6.5+\delta}[1]$ were used in the calculations : (space group $P m m m$ ); $\mathrm{Y}$ at $\left(\frac{1}{2}, \frac{1}{2}, \frac{1}{2}\right), \mathrm{Ba}$ at $\left(\frac{1}{2}, \frac{1}{2}, 0.1874\right)$, $\mathrm{Cu}(1 a)$ at $(0,0,0), \mathrm{Cu}(2 q)$ at $(0,0,0.3565)$. The elements considered for substitution in the $\mathrm{YBa}_{2} \mathrm{Cu}_{3} \mathrm{O}_{6.5+\delta}$ structure were derived either from ionic radii [7] similarities or from literature reports [8-11].

\section{Theoretical background}

For X-ray crystallography the structure factor for any $h k l$ reflection (for "123" structures) can be written as

$$
\begin{aligned}
& F_{h k l}=f_{\mathrm{Y}} \cos 2 \pi\left(h \frac{1}{2}+k \frac{1}{2}+l \frac{1}{2}\right)+2 f_{\mathrm{Ba}} \cos 2 \pi\left(h \frac{1}{2}+k \frac{1}{2}+l 0.1874\right) \\
& +f_{\mathrm{Cu}(1 a)} \cos 2 \pi(h 0+k 0+l 0)+2 f_{\mathrm{Cu}} \cos 2 \pi(h 0+k 0+l 0.3565)+R \\
& =f_{\mathrm{Y}} \cos \pi(h+k+l)+2 f_{\mathrm{Ba}} \cos \pi(h+k+l 0.3748)+f_{\mathrm{Cu}(1 a)} \\
& +2 f_{\mathrm{Cu}(2 q)} \cos \pi(l 0.713)+R .
\end{aligned}
$$

In Eq. (1) $f_{\mathrm{Y}}, f_{\mathrm{Ba}}, f_{\mathrm{Cu}}$ represent atomic scattering factors and $R$ is oxygen contribution to the structure factors. The term $R$ was omitted in initial considerations not only due to relatively small oxygen contribution to the structure factor $(\sim 18 \%$ of the scattering power). First of all the oxygen occupies four different crystallographic positions, and one can predict that particular oxygen atoms contribution can extinct each other. Secondly, cations substitution is relatively easy and perovskite structure is preserved whereas the oxygen atoms substitution caused formation of different compounds. Even though the oxygen term was not written out precisely in formula (1), it was calculated and is presented in Table I. An examination of the atomic scattering factors indicates that the value and sign of $F_{h k l}$ is determined primarily by the barium atoms ( $\sim 39 \%$ of the scattering power), next by the copper atoms $(\sim 10$ and $20 \%)$ and finally by the yttrium atoms $(\sim 13 \%)$.

\subsection{Yttrium substitution}

According to Eq. (1) contribution of the yttrium (or lanthanoid) ion is $+f_{Y}$ for $h+k+l=2 n$ or $-f_{\mathrm{Y}}$ when $h+k+l=2 n+1$. Thus, substitution of a heavier element for $\mathrm{Y}$ can result in an intensity increase or decrease for a particular reflection depending on whether the sum of the remaining components in (1) is negative or positive. 
TABLE I

Contribution of heavy atoms and oxygen to structure factors ${ }^{1,2}$.

\begin{tabular}{|c|c|c|c|c|c|c|c|c|c|c|}
\hline \multicolumn{11}{|c|}{$\mathrm{Cu}$} \\
\hline$l=$ & 0 & 1. & 2 & 3 & 4 & 5 & 6 & 7 & 8 & 9 \\
\hline $\mathrm{Cu}_{2 q}$ & .199 & -.124 & -.046 & .181 & -.178 & .040 & .128 & -.199 & .119 & .051 \\
\hline $\mathrm{Cu}_{1 a+2 q}$ & .299 & -.024 & .054 & .280 & -.079 & .140 & .228 & -.100 & .219 & .151 \\
\hline \multicolumn{11}{|c|}{$\mathrm{Ba}$} \\
\hline$l=$ & 0 & 1 & 2 & 3 & 4 & 5 & 6 & 7 & 8 & 9 \\
\hline$h+k=E^{3}$ & .385 & .148 & -.272 & -.356 & -.001 & .355 & .273 & -.146 & -.385 & -.149 \\
\hline$h+k=O^{3}$ & -.385 & -.148 & .272 & .356 & .001 & -.355 & -.273 & .146 & .385 & .149 \\
\hline \multicolumn{11}{|c|}{$\mathrm{Ba}$ and $\mathrm{Y}$} \\
\hline$l=$ & 0 & 1 & 2 & 3 & 4 & 5 & 6 & 7 & 8 & 9 \\
\hline$h+k=E$ & .519 & .013 & -.138 & -.490 & .133 & .221 & .407 & -.280 & -.251 & -.284 \\
\hline$h+k=O$ & -.519 & -.013 & .138 & .490 & -.133 & -.221 & -.407 & .280 & .251 & .284 \\
\hline \multicolumn{11}{|c|}{$\mathrm{Ba}$ and $\mathrm{Cu}(1 a)+\mathrm{Cu}(2 q)$} \\
\hline$l=$ & 0 & 1 & 2 & 3 & 4 & 5 & 6 & 7 & 8 & 9 \\
\hline$h+k=E$ & .684 & .124 & -.218 & -.076 & -.080 & .496 & .501 & -.245 & -.166 & .002 \\
\hline$h+k=O$ & -.086 & -.172 & .326 & .637 & -.078 & -.215 & -.046 & .046 & .604 & .301 \\
\hline \multicolumn{11}{|c|}{$\mathrm{Cu}$ and $\mathrm{Y}$} \\
\hline$l=$ & 0 & 1 & 2 & 3 & 4 & 5 & 6 & 7 & 8 & 9 \\
\hline$h+k=E$ & .433 & -.158 & .188 & .146 & .056 & .006 & .362 & -.234 & .353 & .017 \\
\hline$h+k=O$ & .165 & .110 & -.080 & .415 & -.213 & .274 & .094 & .034 & .085 & .285 \\
\hline \multicolumn{11}{|c|}{ Heavy atoms contribution } \\
\hline$l=$ & 0 & 1 & 2 & 3 & 4 & 5 & 6 & 7 & 8 & 9 \\
\hline$h+k=E$ & .818 & -.011 & -.084 & -.210 & .055 & .361 & .635 & -.380 & -.032 & -.132 \\
\hline$h+k=O$ & -.220 & -.037 & .192 & .771 & -.212 & -.081 & -.180 & .180 & .470 & .435 \\
\hline \multicolumn{11}{|c|}{ Oxygen contribution } \\
\hline$l=$ & 0 & 1 & 2 & 3 & 4 & 5 & 6 & 7 & 8 & 9 \\
\hline$h=E, k=E$ & .182 & -.033 & .001 & .034 & -.131 & .115 & .051 & -.002 & .122 & -.124 \\
\hline$h=E, k=O$ & .039 & .014 & -.038 & -.071 & -.055 & -.005 & .035 & .028 & -.019 & -.064 \\
\hline$h=O, k=E$ & .072 & .047 & -.005 & -.038 & -.022 & .029 & .068 & .061 & .014 & -.030 \\
\hline$h=O, k=O$ & -.072 & .094 & -.044 & -.142 & .055 & -.091 & .051 & .091 & -.126 & .030 \\
\hline
\end{tabular}

\subsection{Barium substitution}

According to (1) the barium ion contribution depends separately on $l$ and $h+k$. This dependency is summarized in the values of $\cos \pi(h+k+l 0.3748)$ presented below: 


\begin{tabular}{c|r|r|r|r|r|r|c|c|c|c}
\hline \hline$l$ & \multicolumn{1}{c|}{0} & \multicolumn{1}{c|}{1} & \multicolumn{1}{c|}{2} & \multicolumn{1}{c|}{3} & \multicolumn{1}{c|}{4} & \multicolumn{1}{c|}{5} & \multicolumn{1}{c}{6} & \multicolumn{1}{c}{7} & \multicolumn{1}{c}{8} & \multicolumn{1}{c}{9} \\
\hline$h+k=2 n$ & 1 & .383 & -.706 & -.925 & -.003 & .923 & .710 & -.379 & -.999 & -.388 \\
$h+k=2 n+1$ & -1 & -.383 & .706 & .925 & .003 & -.923 & -.710 & .379 & .999 & .388
\end{tabular}

Reflections most sensitive to barium substitution should have $l=0,8,3$, and 5; those least sensitive should have $l=4,7,9$, and 1 . Reflections with $l=0$ are interesting not only because of their high sensitivity to $\mathrm{Ba}$, but also because they are independent of changes in the $z$ coordinate.

\subsection{Copper influence}

Copper occupies two sites: $1 a$ and $2 q$, in space group $P m m m$. The copper atoms in $1 a$ site contribute in any reflection as $+f_{\mathrm{Cu}}$. The influence of $\mathrm{Cu}(2 q)$ site atoms is more complex. From Eq. (1) it is apparent that for any $h \mathrm{kl}$ reflection, the value of $\cos 2 \pi(h x+k y+l z)$ depends only on the $l$ value. This dependency is summarized in the values presented below in the row labeled $\mathrm{Cu}(2 q)$.

\begin{tabular}{c|c|c|c|c|c|c|c|c|c|c}
\hline \hline$l$ & 0 & 1 & 2 & \multicolumn{1}{c|}{3} & \multicolumn{1}{c|}{4} & \multicolumn{1}{c|}{5} & \multicolumn{1}{c|}{6} & \multicolumn{1}{c}{7} & \multicolumn{1}{c}{8} & 9 \\
\hline $\mathrm{Cu}(2 q)$ & 1.0 & -.620 & -.230 & .906 & -.894 & .203 & .642 & -.999 & .598 & .258 \\
$\mathrm{Cu}(1 a)+\mathrm{Cu}(2 q)$ & 1.5 & -.120 & .270 & 1.406 & -.394 & .703 & 1.142 & -.500 & 1.098 & .758
\end{tabular}

The influence of the $\mathrm{Cu}(2 q)$ atoms on the X-ray powder diffraction intensity should be the greatest for reflections with $l=0,7,3,4$ and the smallest for those with $l=2,5,9$.

When both types of copper atoms are considered (random substitution at both $\mathrm{Cu}$ sites is possible), structure factor changes are most pronounced for reflection with a high positive $f_{\mathrm{Cu}(2 q)} \cos \pi(\phi)$ value. (The $\mathrm{Cu}(1 a)$ contribution is always positive and equal to $+f_{\mathrm{Cu}}$.) The sum of the copper site influence on the structure factor is presented in the row labeled $\mathrm{Cu}(1 a)+\mathrm{Cu}(2 q)$. (In this calculation $f_{\mathrm{Cu}}$ is set to 0.5.) Thus, for random copper substitution the most sensitive reflections should be those with $l=0,3,6$. The least sensitive reflections should be those with $l=1$ (nearly completely compensated), 2,4 .

\subsection{Random substitution}

For the random substitution case at two types of positions or by two elements it is useful to consider total scattering power calculated for both positions. Results of such calculations are presented in Table I. Scattering factors $f(\sin \theta / \lambda)$ of considered elements were used as a normalization coefficients. The angular dependence of $f_{i} \sin (\theta / \lambda)$ was not included in the calculation. (The data in Table I can also be useful to predict when the balance of the structure has either a minimum or a maximum influence on a given reflection.) 


\section{Experimental}

Specimens were prepared by mixing appropriate molar proportions of $\mathrm{Y}_{2} \mathrm{O}_{3}$, $\mathrm{Yb}_{2} \mathrm{O}_{3}(99.9 \%$, Research Chemicals, Phoenix, $\mathrm{AZ}), \mathrm{BaCO}_{3}, \mathrm{CuO}$ and $\mathrm{Al}_{2} \mathrm{O}_{3}$ (POCh, Gliwice). Reactans were mixed intimately by grinding under acetone in an agate mortar, then formed into pellets in a mechanic press and heated in air for $36 \mathrm{~h}$ at $950^{\circ} \mathrm{C}$. After slow cooling to ambient temperature at a rate $50^{\circ} \mathrm{C} / \mathrm{h}$, the reaction products were analyzed with Powder Diffractometer DRON-2.0 using filtered $\mathrm{Co} K_{\alpha}$ radiation. X-ray measurements condition: $30 \mathrm{kV}, 20 \mathrm{~mA}, 2 \theta$ range $5-70^{\circ}$, step $0.05^{\circ}$, time for one step $5 \mathrm{~s}$. Sample surfaces dimension: $1.6 \times 1.2 \mathrm{~cm}$. Integrated intensities of diffraction lines were evaluated by the program Newpeak [12]. All products were also examined for the evidence of the Meissner effect by suspending them in a liquid nitrogen in the presence of a strong permanent magnet.

\subsection{Results and discussion}

The mixed metal oxide phases: $\mathrm{YBa}_{2} \mathrm{Cu}_{3} \mathrm{O}_{6.5+x}, \mathrm{Y}_{0.5} \mathrm{Yb}_{0.5} \mathrm{Ba}_{2} \mathrm{Cu}_{3} \mathrm{O}_{6.5+x}$, $\mathrm{YBa}_{2} \mathrm{Cu}_{2.95} \mathrm{Al}_{0.05} \mathrm{O}_{6.5+x}$ and $\mathrm{YBa}_{2} \mathrm{Cu}_{2.85} \mathrm{Al}_{0.15} \mathrm{O}_{6.5+x}$ were synthesized. All these phases but the last one show apparence of the Meissner effect in the liquid nitrogen temperature. The X-ray powder diffraction patterns indicate the pure "123"-type perovskite related phases, with intensities strongly affected by preferred orientation (along [001]). To minimize the preferred orientation errors and to prove the substitution, only the intensities of a few nonoverlaping $(00 l)$ reflections were considered. According to brief calculations presented in Table I, or calculations performed using the LAZY-PULVERIX program one can predict that with substitution of $Y$ by heavier $\mathrm{Yb}$ element, intensities of (001) and (003) lines should increase, while intensities of (002) and (004) lines should decrease. In the pure YBaCu HTSC intensities of (001), (002), (003) and (004) lines are 12, 23,60,6\% while in $\mathrm{YYbBaCu}$ sample are $43,7,75$ and $2.5 \%$, respectively (intensity of (006) reflection was taken as $100 \%$ ). Thus, the data presented above clearly indicate the substitution of $\mathrm{Y}$ by $\mathrm{Yb}$ atoms.

The situation is more complicated in the case of $\mathrm{Cu}$ (at $0001 a$ position) substituted by Al. Since contribution of $1 a$ position to structure factor is always positive, and total structure factors of $\mathrm{Ba}, \mathrm{Y}$, and $\mathrm{Cu}(2 q)$ for reflections selected above are negative, copper substitution by $\mathrm{Al}$ should result in increase of all these reflections. It should be most pronounced in the case of (001) due to large LP (Lorenz polarization correction) value, less in the case of (002), (003) and (004). Indeed intensity of (001) line for samples with 0,5 and $15 \%$ of $\mathrm{Al}$ are 11.5, 18.8 and $21.8 \%$, clearly indicating substitution (average error $<1 \%$ ). Intensities of another lines, as could be predicted, do not confirm substitution so unequivocally. Situation should be much better when a bigger group of reflections will be considered and first of all after preferred orientation elimination. 


\section{Comparison with literature data}

\subsection{X-ray diffraction}

Franck et al. [3] present an X-ray diffractogram of a few reflections of $\mathrm{Y}_{1-x} \mathrm{Al}_{x} \mathrm{Ba}_{2} \mathrm{Cu}_{3} \mathrm{O}_{6.5}$ phase for $x=0.2,0.5,0.7$ and 1.0 , and presented conclusions based primarily upon reflection positional changes. For the $x=1.0$ diffractogram reflections typical of the " 123 "-type phase have almost disappeared and a new set of strong reflections is present, suggestive that the specimen is predominantly a different phase with a trace amount of the " 123 "-type of structure. More detailed descriptions of $(110),(013)(d$-values $=2.725 \AA)$, and $(013)(d$-value $=2.75 \AA)$ reflections are presented separately. For these three reflections $h+k+l=2 n$ and the $Y$-site contribution to the structure factor is $+f_{Y}$. The contributions of every other cation site to the structure factor are also positive, and the 103 and 013 forms of the structure factor are identical. These reflections are among the strongest in the diffraction pattern and consequently are insensitive to substitution, even substitution at a different cation site. All of them should increase or decrease simultaneously. (Substitution of Al up to $x=0.22$ was subsequently demonstrated to occur only at the Cu-site [13].)

Somasundaram et al. [14] also reported some X-ray powder data for $\mathrm{YBa}_{2-x} \mathrm{La}_{x} \mathrm{CuO}_{7+y}$ and presented conclusions based upon positional changes. The scattering power of $\mathrm{Ba}^{2+}$ and $\mathrm{La}^{3+}$ are almost identical; both ions have the same number of electrons. The intensities of reflections (113), (104) and (105) are presented. Our data (presented in Table I) indicate that the intensity of reflection (104) is essentially independent of the ion occupying the Ba-site; its contribution to the structure factor is $0.006 f_{\mathrm{Ba}}$. Reflections (005) and (113), on the other hand, do have a strong Ba-site contribution. Therefore, the observed intensity variation of (104) and (005), respectively, as equal, smaller, greater, smaller, smaller, greater and smaller than the intensity of (113) for $x=0,0.125,0.25,0.325,0.35,0.375$ and 0.50 cannot result from substitution. The changes are more probably caused by impurities or texture variations.

\subsection{Neutron diffraction}

Neutron diffraction experiments are particularly useful when oxygen atoms position, oxygen substitution or vacancies, substitution of $\mathrm{Ni}$, Co or $\mathrm{Zn}$ for $\mathrm{Cu}$ atoms are being investigated. Considerations similar to those presented above can help to decide whether such, rather expensive and time consuming experiments will solve the problem. Recently chemical, structural and electrical properties of fluoride treated $\mathrm{YBa}_{2} \mathrm{Cu}_{3} \mathrm{O}_{6.5}$ were reported [11]. On the basis of powder X-ray and chemical analysis the authors concluded that $\mathrm{F}$ anions had been incorporated chemically, but their position was not determined. Fluoride has only one more electron than oxygen and cannot be located by X-ray diffraction in the presence of heavy atoms. In this case neutron diffraction would seem to provide the solution. However, the neutron scattering lengths for $O$ and F, 0.580 and $0.566 \times 10^{-12} \mathrm{~cm}$, respectively, suggest problems as serious as those encountered by X-ray diffraction. 
It should be relatively easy to determine sulfur substitution for oxygen atoms by neutron diffraction because the difference in scattering lengths of $O$ and $S$, 0.580 and $0.285 \times 10^{-12} \mathrm{~cm}$, respectively, is large. By calculating the oxygen and heavy atom individual contributions to the neutron diffraction pattern, reflections with the highest oxygen and minimal heavy atom contribution could be selected. Changes of intensities of (001), (100), (110), (113), (024), and (204) reflections should provide a good indication of oxygen substitution or of vacancies in oxygen positions.

\subsection{Real situation, limitations, conclusions}

In general, (normalized) intensities are measured (or reported), not structure factors. Resolution of reflections can also be a problem. Perovskite related structures possess groups of strong reflections with similar $d$-spacing, e.g. (006), (200), and (020) or (003), (100), (010), so the intensity estimation can be difficult. The Rietveld procedure overcomes these limitations as the full pattern fitting programs usually can extract the appropriate information even from non-ideal diffraction data. With "integrated intensity data" it would be useful to calculate the X-ray powder pattern for a few artificial structures based on an initial structure from which some elements have been neglected. Considering intensities and structure factors with their sign, as reported by the program LAZY-PULVERIX [15], one can find the set of reflections suitable to verify the investigated hypothesis. We hope the considerations presented here will help investigators select powder diffraction reflections that are sensitive to intensity as well as positional variation in their experiments to help them arrive at correct conclusions. Information about substitution in "123"-type compounds can indeed be determined by powder diffraction, but reflections examined must be chosen carefully.

\section{Acknowledgements}

Financial support provided by the Polish Research Council (KBN) and support of the National Science Foundation, Division of Material Science, Solid State Chem. Program DMR 84-00739 is acknowledged gratefully.

\section{References}

[1] Y. Le Page, T. Siegrist, S.A. Sunshine, L.F. Schneemeyer, D.W. Murphy, S.N. Zahurak, J.V. Waszczak, W.R. McKinnon, J.M. Tarascon, G.W. Hull, L.H. Greene, Phys. Rev. B 36, 3617 (1987).

[2] J. Jung, J.P. Franc, W.A. Miner, M.A.-K. Mohamed, Phys. Rev. B 37, 7510 (1988).

[3] J.P. Franck, J. Jung, M.A.-K. Mohamed, Phys. Rev. B 36, 2308 (1987).

[4] S.A. Hodorowicz, W. Łasocha, A. Easocha, H.A. Eick, J. Solid State Chem. 77, 148 (1988).

[5] M. Izumi, T. Yabe, T. Wada, A. Maeda, K. Uchinokura, H. Asano, Phys. Rev. B 40, 6771 (1989).

[6] Y. Xu, R.L. Sabatini, A.R. Moodenbaugh, Y. Zhu, S.-G. Shyu, M. Suenaga, K.W. Dennis, R.W. McCallum, Physica C 169, 205 (1990). 
[7] R.D. Shannon, Acta Crystallogr. A 32, 751 (1976).

[8] A.F. Hepp, J.R. Gaier, P.D. Hambourger, J.J. Pouch, A. Banerjea, Superc. its Appl. Proc. Annu. Conf. Superc. Appl. 2nd, 146 (1988).

[9] M.M. Michael, N.A. Mukhlif, Mater. Res. Bull. 23, 1797 (1988).

[10] Y. Matsumoto, J. Hombo, Y. Yamaguchi, Mater. Res. Bull. 24, 1231 (1989).

[11] H.H. Wang, A.M. Kini, H.-C.I. Kao, E.H. Appleman, A.R. Thompson, R.E. Botto, K.D. Carlson, J.M. Williams, M.Y. Chen, J.A. Schlueter, B.D. Gates, S.L. Hallenbeck, A.M. Despotes, Inorg. Chem. 27, 5 (1988).

[12] E.J. Sonnefeld, J.W. Visser, J. Appl. Cryst. 8, 1 (1975).

[13] T. Siegrist, L.F. Schneemeyer, J.V. Waszczak, N.P. Singh, R.L. Opila, B. Batlogg, L.W. Rupp, D.W. Murphy, Phys. Rev. B 36, 8365 (1987).

[14] P. Somasundaram, K.S. Nanjundaswamy, A.M. Umarji, C.N.R. Rao, Mater. Res. Bull. 23, 1139 (1988).

[15] K. Yvon, W. Jeitschko, E. Parthe, J. Appl. Cryst. 10, 73 (1977). 